Bull. Korean Math. Soc. 51 (2014), No. 2, pp. 531-538

http://dx.doi.org/10.4134/BKMS.2014.51.2.531

\title{
A CHARACTERIZATION OF CONCENTRIC HYPERSPHERES IN $\mathbb{R}^{n}$
}

\author{
Dong-Soo Kim and Young Ho Kim
}

\begin{abstract}
Concentric hyperspheres in the $n$-dimensional Euclidean space $\mathbb{R}^{n}$ are the level hypersurfaces of a radial function $f: \mathbb{R}^{n} \rightarrow \mathbb{R}$. The magnitude $\|\nabla f\|$ of the gradient of such a radial function $f: \mathbb{R}^{n} \rightarrow \mathbb{R}$ is a function of the function $f$. We are interested in the converse problem. As a result, we show that if the magnitude of the gradient of a function $f: \mathbb{R}^{n} \rightarrow \mathbb{R}$ with isolated critical points is a function of $f$ itself, then $f$ is either a radial function or a function of a linear function. That is, the level hypersurfaces are either concentric hyperspheres or parallel hyperplanes. As a corollary, we see that if the magnitude of a conservative vector field with isolated singularities on $\mathbb{R}^{n}$ is a function of its scalar potential, then either it is a central vector field or it has constant direction.
\end{abstract}

\section{Introduction}

Consider a radial function $f: \mathbb{R}^{n} \rightarrow \mathbb{R}$, that is, $f$ satisfies $f(x)=g(\|x\|)$ for some function $g$. Then it is well-known that the magnitude of the gradient of $f$ is a function of the function $f$.

In this regard, for a function $f: \mathbb{R}^{n} \rightarrow \mathbb{R}$, we consider the following condition

$$
\|\nabla f(p)\|=\phi(f(p)), \quad p \in \mathbb{R}^{n},
$$

where $\phi: \mathbb{R} \rightarrow[0,+\infty)$ is a real valued function. Then, the radial functions on $\mathbb{R}^{n}$ satisfy condition $(\mathrm{C})$

Therefore, it is natural to ask a question:

"What kinds of functions on $\mathbb{R}^{n}$ satisfy condition (C)?"

Received December 24, 2012

2010 Mathematics Subject Classification. 53A07.

Key words and phrases. gradient, conservative vector field, central vector field, hypersurface, principal curvature, radial function.

The first author was supported by Basic Science Research Program through the National Research Foundation of Korea (NRF) funded by the Ministry of Education, Science and Technology (2010-0022926).

The second author was supported by Basic Science Research Program through the National Research Foundation of Korea (NRF) funded by the Ministry of Education, Science and Technology (2012R1A1A2042298). 
In this article, we study the functions defined on $\mathbb{R}^{n}$ which satisfy condition (C). As a result, first, we establish a local characterization of functions satisfying condition $(\mathrm{C})$.

Proposition 1. For a function $f$ defined on an open set $V \subset \mathbb{R}^{n}$ without critical points, the following are equivalent:

1) The function $f$ satisfies condition $(\mathrm{C})$.

2) Every integral curve of $\nabla f$ is a straight line.

3) For a level hypersurface $M, f$ is constant on each parallel hypersurface of $M$.

Second, using Proposition 1, we prove the following characterization theorem of functions satisfying condition (C) globally on $\mathbb{R}^{n}$.

Theorem 2. Suppose that a function $f: \mathbb{R}^{n} \rightarrow \mathbb{R}$ with isolated critical points satisfies condition (C). Then $f$ is a function of either a distance function $r=$ $\|p-o\|$ from a fixed point o or a linear function. That is, the level sets are either concentric hyperspheres or parallel hyperplanes.

As applications of Theorem 2, we get the following characterizations.

Corollary 3. Suppose that a function $f$ defined on $\mathbb{R}^{n}$ with isolated critical points satisfies condition $(\mathrm{C})$. Then we have the following.

1) If $f$ has no critical points, then $f$ is a function of some linear function.

2) If $f$ has at least one critical point, then $f$ is a radial function from a fixed point $o$.

In particular, we have:

Corollary 4. For a function $f$ defined on $\mathbb{R}^{n}$, the following are equivalent:

1) The magnitude $\|\nabla f\|$ of the gradient of $f$ is constant on $\mathbb{R}^{n}$.

2) The function $f$ is a linear function.

It follows from Corollary 4 that a conservative vector field with constant magnitude defined on the whole space $\mathbb{R}^{n}$ is a constant vector field.

For a conservative vector field $F$ on $\mathbb{R}^{n}$, condition $(\mathrm{C})$ is equivalent to the following:

"The magnitude of a conservative vector field $F$ is a function of its scalar potential function."

For a fixed point $o \in \mathbb{R}^{n}$, a vector field $F$ defined on $\mathbb{R}^{n} \backslash\{o\}$ is called a central vector field if it is invariant under orthogonal transformations around $o$. The point $o$ is called the center of the vector field $F$. The gradient vector field of a radial function from a point $o \in \mathbb{R}^{n}$ is an example of such vector fields.

Since orthogonal transformations around a fixed point $o$ are actually rotations and reflections, the invariance conditions show that vectors of a central vector field are always directed towards, or away from, its center $o$. Hence, it is straightforward to show that every central vector field with center $o \in \mathbb{R}^{n}$ is the gradient vector field of a radial function from $o$. 
Now, we may restate Theorem 2 as follows.

Theorem 5. Suppose that the magnitude of a conservative vector field $F$ on $\mathbb{R}^{n}$ with isolated singularities is a function of its scalar potential function. Then we have the following.

1) If $F$ has no singularities, then $F$ has constant direction.

2) If $F$ has at least one singularity, then $F$ has exactly one zero o $\in \mathbb{R}^{n}$ and $F$ is a central vector field with center $o$.

Theorem 2 or Theorem 5 might be well-known, but we could not find the references for them (cf. [7]).

Throughout this article, all objects are smooth (that is, $C^{2}$ ), unless otherwise mentioned. For notations and terminologies, see [2], [3], [5] or [9].

\section{Proofs}

Suppose that $\|\nabla f\|=\phi(f)$ for a real valued function $\phi$. Let $R_{f}$ denote the set of regular values of $f$. For $k \in R_{f}$, let's denote by $U$ the unit normal vector to the level hypersurface $f^{-1}(k)$ in the direction of $\nabla f$. Hence, it follows from condition (C) that

$$
\nabla f=\phi(k) U \quad \text { on } \quad f^{-1}(k) .
$$

First, we prove the following lemma.

Lemma 6. For a function $f$ defined on an open set $V \subset \mathbb{R}^{n}$ without critical points, the following are equivalent:

1) $\|\nabla f\|=\phi(f)$ for a function $\phi$.

2) Every integral curve $y(t)$ of $\nabla f$ is a straight line.

Proof. For a point $p \in f^{-1}(k)$ with $k \in R_{f}$, we denote by $\kappa_{1}(p), \ldots, \kappa_{n-1}(p)$ the principal curvatures of the level hypersurface $f^{-1}(k)$ associated with the corresponding principal directions $e_{1}(p), \ldots, e_{n-1}(p)$ with respect to $U$ at $p$.

For each $i=1,2, \ldots, n-1$, we let $x_{i}(s)$ denote a unit speed curve of $f^{-1}(k)$ starting from $p$ with $x_{i}^{\prime}(0)=e_{i}(p), i=1,2, \ldots, n-1$. Then (1) implies for each $i=1,2, \ldots, n-1$

$$
\nabla f\left(x_{i}(s)\right)=\phi(k) U\left(x_{i}(s)\right) .
$$

By differentiating (2) with respect to $s$, we get at $s=0$

$$
H^{f}(p) e_{i}(p)=-\phi(k) \kappa_{i}(p) e_{i}(p),
$$

where $H^{f}$ denotes the Hessian matrix of $f$. Since $H^{f}(p)$ is symmetric, from (3) we see that for any point $p \in f^{-1}(k)$

$$
H^{f}(p) \nabla f(p)=h(p) \nabla f(p),
$$

where $h$ is a function. It follows from (4) that for all $x \in \mathbb{R}^{n}, f$ satisfies

$$
H^{f}(x) \nabla f(x)=h(x) \nabla f(x) .
$$


Let's denote by $y(t)$ the integral curve of $\nabla f$ with $y(0)=p \in f^{-1}(k)$. Then we have

$$
y^{\prime \prime}(t)=H^{f}(y(t)) \nabla f(y(t))=h(t) \nabla f(y(t))=h(t) y^{\prime}(t)
$$

where $h(t)=h(y(t))$ and the second equality follows from (5). This shows that $y(t)$ is a parametrization of a straight line.

Conversely, suppose that every integral curve $y(t)$ of $\nabla f$ is a straight line. Then every integral curve $y(t)$ satisfies $y^{\prime \prime}(t)=h(t) y^{\prime}(t)$ for some function $h$. Hence (6) shows that $f$ satisfies (5) for all $x \in \mathbb{R}^{n}$.

Now, for a fixed unit speed curve $x(s)$ on the level hypersurface $f^{-1}(k)$, we differentiate $\|\nabla f(x(s))\|^{2}$ as follows.

$$
\begin{aligned}
\frac{d}{d s}\|\nabla f(x(s))\|^{2} & =2\left\langle\frac{d}{d s} \nabla f(x(s)), \nabla f(x(s))\right\rangle \\
& =2\left\langle H^{f}(x(s)) x^{\prime}(s), \nabla f(x(s))\right\rangle \\
& =2\left\langle x^{\prime}(s), H^{f}(x(s)) \nabla f(x(s))\right\rangle \\
& =2\left\langle x^{\prime}(s), h(x(s)) \nabla f(x(s))\right\rangle \\
& =0
\end{aligned}
$$

where the 3rd and 4th equalities follow from the symmetry of $H^{f}$ and (5), respectively. Thus, $\|\nabla f\|^{2}$ is constant on each level hypersurface of $f$. This completes the proof.

For a hypersurface $M$ of $\mathbb{R}^{n}$ with a unit normal vector field $U$, parallel hypersurfaces $M_{t}, t \in \mathbb{R}$ of $M$ are defined by

$$
M_{t}=\{p+t U(p) \mid p \in M\} .
$$

Next, we show that the level hypersurfaces of $f$ are parallel.

Lemma 7. Suppose that a function $f$ defined on an open set $V \subset \mathbb{R}^{n}$ without critical points satisfies condition (C). Then, for a level hypersurface $M$ of $f, f$ is constant on each parallel hypersurface of $M$.

Proof. Let $x(s)$ denote a fixed unit speed curve of a level hypersurface $f^{-1}(k)$ of $f$. We consider the integral curve $y_{s}(t)$ of $\nabla f$ with $y_{s}(0)=x(s)$. Then, from Lemma 6 we have

$$
y_{s}(t)=x(s)+a(t) \nabla f(x(s)),
$$

where $a(t)$ is a function with $a(0)=0$. Since $\nabla f(x(s))=y_{s}^{\prime}(0)=a^{\prime}(0) \nabla f(x(s))$, we get $a^{\prime}(0)=1$. 
By differentiating $f\left(y_{s}(t)\right)$ with respect to $s$, it follows from (5) and (8) that

$$
\begin{aligned}
\frac{d}{d s} f\left(y_{s}(t)\right) & =\left\langle\nabla f\left(y_{s}(t)\right), \frac{d}{d s} y_{s}(t)\right\rangle \\
& =\left\langle\nabla f\left(y_{s}(t)\right), x^{\prime}(s)+a(t) H^{f}(x(s)) x^{\prime}(s)\right\rangle \\
& =a(t) a^{\prime}(t)\left\langle\nabla f(x(s)), H^{f}(x(s)) x^{\prime}(s)\right\rangle \\
& =a(t) a^{\prime}(t)\left\langle H^{f}(x(s)) \nabla f(x(s)), x^{\prime}(s)\right\rangle \\
& =0 .
\end{aligned}
$$

Hence $f\left(y_{s}(t)\right)$ is a function of $t$ only. This shows that $f$ is constant on each parallel hypersurface of $M$.

If we let $k(t)=f\left(y_{s}(t)\right)$, then we have from condition $(\mathrm{C})$

$$
k^{\prime}(t)=\left\langle\nabla f\left(y_{s}(t)\right), \nabla f\left(y_{s}(t)\right)\right\rangle=\phi(k(t))^{2}, \quad k(0)=k .
$$

On the other hand, from (8) we get

$$
k^{\prime}(t)=\left\langle y_{s}^{\prime}(t), y_{s}^{\prime}(t)\right\rangle=\phi(k)^{2} a^{\prime}(t)^{2} .
$$

It follows from (10) and (11) that

$$
\phi(k(t))=\phi(k) a^{\prime}(t) .
$$

Thus $a(t)$ is determined by (12) with $a(0)=0$, which is independent of $x(s)$.

Remark 8. If we let $z_{s}(t)$ denote the integral curve of $U=\nabla f /\|\nabla f\|$ with $z_{s}(0)=x(s)$, then we have $z_{s}(t)=x(s)+t U(x(s))$ and $\frac{d}{d s} f\left(z_{s}(t)\right)=0$. For $k(t)=f\left(z_{s}(t)\right)$, we get $k^{\prime}(t)=\phi(k(t))$.

Conversely, suppose that $f$ is constant on each parallel hypersurface $M_{t}$. That is, $f(p+t U(p))=k(t), p \in M$, where $k(t)$ is a function of $t$. Then we have

$$
\nabla f(p+t U(p))=k^{\prime}(t) U(p) .
$$

Hence $f$ satisfies condition (C) with $\phi= \pm k^{\prime} \circ k^{-1}$ if $k^{\prime}(t) \neq 0$.

Thus, we have the following local characterization of functions satisfying condition (C).

Proposition 1. For a function $f$ defined on an open set $V \subset \mathbb{R}^{n}$ without critical points, the following are equivalent:

1) $f$ satisfies condition $(\mathrm{C})$.

2) Every integral curve of $\nabla f$ is a straight line.

3) For a level hypersurface $M, f$ is constant on each parallel hypersurface of $M$.

Now, we prove the main theorem as follows. 
Theorem 2. Suppose that a function $f: \mathbb{R}^{n} \rightarrow \mathbb{R}$ with isolated critical points satisfies condition $(\mathrm{C})$. Then $f$ is a function of either a distance function $r=$ $\|p-o\|$ from a fixed point o or a linear function. That is, the level sets are either concentric hyperspheres or parallel hyperplanes.

Proof. Suppose that a function $f: \mathbb{R}^{n} \rightarrow \mathbb{R}$ with isolated critical points satisfies condition (C) globally on $\mathbb{R}^{n}$. Without loss of generality, we may assume that $0 \in R_{f}$. Then, the above discussion shows that for the unit normal $U=\nabla f /\|\nabla f\|$ to the level hypersurface $M_{0}=f^{-1}(0)$, the function $f$ and $\nabla f$ is given by

$$
f(p+t U(p))=k(t) \quad \text { and } \quad \nabla f(p+t U(p))=k^{\prime}(t) U(p),
$$

where $k(t)$ is a function with $k(0)=0$ and $k^{\prime}(0) \neq 0$.

We consider the flow $y: M_{0} \times \mathbb{R} \rightarrow \mathbb{R}^{n}$ given by

$$
y(p, t)=p+t U(p) .
$$

For a point $p \in M_{0}$, we denote by $\kappa_{1}(p), \ldots, \kappa_{n-1}(p)$ the principal curvatures of $M$ associated with the corresponding principal directions $e_{1}(p), \ldots, e_{n-1}(p)$ with respect to $U$ at $p$. For each $i=1,2, \ldots, n-1$, we let $x_{i}(s)$ denote a unit speed curve of $M_{0}$ starting from $p$ with $x_{i}^{\prime}(0)=e_{i}(p), i=1,2, \ldots, n-1$. Then, we have for each $i=1,2, \ldots, n-1$

$$
\left.\frac{d}{d s} y\left(x_{i}(s), t\right)\right|_{s=0}=\left(1-t \kappa_{i}(p)\right) e_{i}(p) .
$$

Since $k^{\prime}(0) \neq 0$, we see that $k^{\prime}(t) \neq 0$ on an open interval $I_{0}$ containing 0 . Hence for each $t \in I_{0}$, the level set $M_{t}=f^{-1}(k(t))$ is a nonsingular hypersurface. Consider an open interval $I$ containing 0 . Then it follows from (15) and (16) that the level sets $M_{t}, t \in I$ are all nonsingular hypersurfaces if and only if each $t \in I$ satisfies the following

$$
1-t \kappa_{i}(p)>0, i=1,2, \ldots, n-1 \text { and } p \in M_{0} .
$$

Let's denote by $I^{*}$ the maximal open interval containing 0 such that each $t \in I^{*}$ satisfies (17).

First, suppose that the maximal interval $I^{*}$ has an end point $t_{0}$. Then we have $k^{\prime}\left(t_{0}\right)=0$, otherwise $t_{0}$ is contained in $I^{*}$. Hence every point of the level set $M_{t_{0}}$ is a critical point of the function $f$. Since such points are isolated, we see that the level set $M_{t_{0}}$ is a fixed point $o \in \mathbb{R}^{n}$.

This shows that $M_{0}$ is a hypersphere of radius $\left|t_{0}\right|$ centered at $o$. Hence we have $\kappa_{i}(p)=1 / t_{0}$ for all $i=1,2, \ldots, n-1$ and $p \in M_{0}$. It follows from (17) that $I^{*}=\left(t_{0}, \infty\right)$ or $I^{*}=\left(-\infty, t_{0}\right)$ according to the sign of $t_{0}$. Since each level set $M_{t}$ of $f$ is a parallel hypersurface of $M_{0}$, it is also a hypersphere centered at $o$. Therefore $f$ is a function of the distance function $r=\|p-o\|$ from the point $o$. Thus, $f$ is a radial function from the point $o$. 
Finally, suppose that the maximal interval $I^{*}$ is the real line $\mathbb{R}$. Then, it follows from (17) and the definition of $I^{*}$ that

$$
\kappa_{i}(p)=0, i=1,2, \ldots, n-1 \text { and } p \in M_{0} .
$$

Therefore $M_{0}$ is a hyperplane, and hence every level set of $f$ is also a hyperplane. Thus $f$ is a function of a linear function. This completes the proof of Theorem 2 .

From Theorem 2, immediately we get Corollaries 3, 4 and Theorem 5 .

Remark. Suppose that a function $f: \mathbb{R}^{n} \rightarrow \mathbb{R}$ with isolated critical points satisfies condition (C) for some $\phi: \mathbb{R} \rightarrow[0,+\infty)$.

If we additionally assume that $f$ has at most one critical value (say, $f(p)=a$, and hence we have $\phi(a)=0)$, then setting $h(r):=\int_{r_{0}}^{r} \phi(s)^{-1} d s\left(\left[r_{0}, r\right]\right.$ being a segment outside $\left.\phi^{-1}(0)=\{a\}\right)$, we obtain $\|\nabla(h \circ f)(x)\|=1$ on a domain in $\mathbb{R}^{n}$. This is the classical eikonal equation (cf. [4] and [8]). But, the solutions of eikonal equations do not imply our theorems in this article.

Instead, in case $f$ has no critical points, $h \circ f$ is a function globally defined on $\mathbb{R}^{n}$ with $\|\nabla(h \circ f)(x)\|=1$. Hence $h \circ f$ is a linear function (Remark 2.3 of [1] and [6]). This gives a proof of 1) in Corollary 3.

\section{References}

[1] L. A. Caffarelli and M. G. Crandall, Distance functions and almost global solutions of eikonal equations, Comm. Partial Differential Equations 35 (2010), no. 3, 391-414.

[2] M. P. do Carmo, Differential Geometry of Curves and Surfaces, Prentice-Hall, Englewood Cliffs, NJ, 1976.

[3] T. L. Chow, Mathematical Methods for Physicists: A concise introduction, Cambridge University Press, Cambridge, 2000.

[4] R. Courant and D. Hilbert, Methods of Mathematical Physics. Vol. II, Partial differential equations, Reprint of the 1962 original, Wiley Classics Library, A Wiley-Interscience Publication, John Wiley and Sons, Inc., New York, 1989.

[5] M. W. Hirsch and S. Smale, Differential equations, dynamical systems, and linear algebra, Pure and Applied Mathematics, Vol. 60, Academic Press, New York-London, 1974.

[6] D. Khavinson, A note on entire solutions of the eiconal [eikonal] equation, Amer. Math. Monthly 102 (1995), no. 2, 159-161.

[7] K. Nomizu, Elie Cartan's work on isoparametric families of hypersurfaces, Differential geometry (Proc. Sympos. Pure Math., Vol. XXVII, Part 1, Stanford Univ., Stanford, Calif., 1973), pp. 191-200, Amer. Math. Soc., Providence, R.I., 1975.

[8] O. N. Stavroudis and R. C. Fronczek, Caustic surfaces and the structure of the geometrical image, J. Opt. Soc. Amer. 66 (1976), no. 8, 795-800.

[9] J. A. Thorpe, Elementary Topics in Differential Geometry, Undergraduate Texts in Mathematics, Springer-Verlag, New York-Heidelberg, 1979.

DONG-SOO KIM

Department of Mathematics

Chonnam National University

KWANGJU 500-757, Korea

E-mail address: dosokim@chonnam.ac.kr 
YOUNG Ho KIM

Department of Mathematics

KYUngPoOK NATIONAL University

TAEGU 702-701, KoREA

E-mail address: yhkim@knu.ac.kr 